\title{
Pharmacokinetics in morbid obesity: influence of two bariatric surgery techniques on paracetamol and caffeine metabolism
}

Purpose: To study the impact of the two most common bariatric surgery techniques on paracetamol pharmacokinetics (a marker of gastric emptying) and caffeine metabolism (a marker of liver function).

Materials and methods: In the present prospective study, we studied 24 morbid obese patients before, at 4 weeks, and 6 months after having undergone sleeve gastrectomy $(n=10)$ or Roux-en-Y gastric bypass $(n=14)$. For comparative purposes, 28 healthy controls (14 normal weight and 14 overweight) were also included in the study.

Results: Paracetamol pharmacokinetics was altered in the obese participants leading to lower bioavailability. Bariatric surgery resulted in faster absorption and normalized pharmacokinetic parameters, prompting an increase in paracetamol bioavailability. No differences were found between surgical procedures. In the case of caffeine, the ratio paraxanthine/caffeine did not differ between morbid obese and healthy individuals. This ratio remained unmodified after surgery, indicating that liver function (assessed by cytochrome P450 1A2 activity) was unaffected by obesity or bariatric surgery.

Conclusions: Paracetamol pharmacokinetics and caffeine plasma levels are altered in severely obese patients. The two studied bariatric surgical techniques normalize paracetamol oral bioavailability without impairing liver function (measured by cytochrome P450 1A2 activity). 
Keywords: Clinical trial; gastric bypass; sleeve gastrectomy; morbid obesity; metabolism; paracetamol; caffeine.

\section{Introduction/Purpose}

Bariatric surgery involves a considerable alteration of the gastrointestinal tract because absorption is reduced and/or the absolute volume of the material that can be ingested at one time is restricted. Despite the beneficial effects of bariatric surgery on severely obese patients, surgical-derived alterations may have an impact on drug absorption and bioavailability that will ultimately lead to consequences regarding dosage and administration [1]. To date, predicting the impact of bariatric surgery on drug pharmacokinetics constitutes a research challenge and it is considered to be drug-specific [2, 3].

In this work, we report the impact of the two most common bariatric surgery techniques (laparoscopic Roux-en-Y gastric bypass (LRYGB) and Laparoscopic Sleeve Gastrectomy (LSG)) on paracetamol pharmacokinetics and caffeine metabolism. Paracetamol (acetaminophen), the most commonly used non-opioid analgesic, and caffeine were chosen to study how gastric emptying and liver function are altered by bariatric surgery, respectively. In the case of paracetamol, its absorption is negligible in the stomach but very rapid from the small intestine, the rate of absorption, therefore, depends on the rate of gastric emptying [4-7]. With respect to caffeine, the measurement of caffeine concentration in a single plasma sample has been proposed as a simple guide to evaluate liver function [8]. Moreover, the concentration of the molar ratio paraxanthine/caffeine $(17 \mathrm{X} / 137 \mathrm{X})$ in plasma assessed by one sampling point taken 4 hours post dose is a straightforward, reliable, and relatively inexpensive estimate of cytochrome P450 1A2 (CYP1A2) activity [9] as it resembles systemic caffeine clearance [10]. 


\section{Materials and Methods}

\section{Subjects and study protocol}

A prospective cohort study was conducted in 24 patients with severe obesity planning to undergo bariatric surgery at Hospital del Mar (Barcelona, Spain). They were between 18 and 55 years of age and met the 1991 bariatric surgery criteria of the National Institutes of Health [11]. Two different surgical procedures were considered: LRYGB $(n=14)$ and LSG $(n=10)$. Indication for the type of surgical procedure was based on clinical criteria and the consensus of the Bariatric Surgery Unit as previously described $[12,13]$. Sample size was calculated to detect (1) a difference of at least a $30 \%$ in the depuration of the drugs used as biomarkers of a metabolic activity between obese subjects and controls, and (2) a 50\% modification of paracetamol absorption velocity with a statistical power of 0.8 . Using two-tailed tests and accepting an alpha risk of 0.05 , a total number of 12 controls, 12 obese patients undergoing LSG and 12 obese patients undergoing LRYGB were considered necessary.

We administered paracetamol and caffeine to obese patients before and at 4 weeks and 6 months after two different bariatric surgery techniques. For comparative purposes, paracetamol pharmacokinetics and caffeine metabolism were also evaluated in 14 overweight subjects and 14 normal weight control subjects. Protocol appointments included measurements of weight, waist and hip circumferences, as well as pharmacokinetic measurements.

Participants were instructed not to consume ethanol, caffeine, or xanthine-related products (e.g. coffee, tea, colas, chocolate) for 2 days before the study sessions. Subjects were excluded if they were taking medication known to induce or inhibit CYP enzymes or had a history of hypersensitivity to drugs used in the study. After overnight fasting and baseline procedures, each subject received a modified Karolinska cocktail $[14,15]$ including 5 drugs: 
dextromethorphan (Romilar $\left.{ }^{\circledR}, 30 \mathrm{mg}\right)$, losartan (Cozaar $\left.{ }^{\circledR}, 12.5 \mathrm{mg}\right)$, omeprazole (Losec $®, 20$ $\mathrm{mg}$ ), caffeine $(65 \mathrm{mg})$, and paracetamol (Panadol® Extra, containing $500 \mathrm{mg}$ of paracetamol and $65 \mathrm{mg}$ of caffeine). Dextromethorphan $(30 \mathrm{mg}$ ) was self-administered the night before each session. Losartan (25 mg), paracetamol-caffeine (500 mg and $65 \mathrm{mg}$ ) were administered between 08:00 and 08:15 hours and omeprazole (20 mg) one hour later.

Blood samples ( $3 \mathrm{~mL}$ each) were taken at: 0, 15, 30, 60 minutes and 2, 3 and 4 hours after oral cocktail administration for paracetamol analysis and at 0 and 4 hours for caffeine analysis. Urine was collected overnight (0-8 hours) after dextromethorphan and during the experimental session. For comparative purposes, 14 overweight subjects (BMI $25-30 \mathrm{~kg} / \mathrm{m}^{2}$ ), and 14 normal weight control subjects $(\mathrm{BMI}<25)$ were additionally included in the study and also received the same modified Karolinska cocktail. This procedure was performed a single time in healthy volunteers (overweight and normal weight control subjects) and three times is MO patients: before surgery, 4 weeks after surgery, and 6 months after surgery.

All MO subjects were informed of the surgical risks and benefits and signed their informed consent for the surgical procedure. All subjects also signed an informed consent document for study participation.

\section{Surgical techniques}

The LRYGB technique involved a 150-cm antecolic Roux limb with 25-mm circular pouch-jejunostomy and exclusion of $50 \mathrm{~cm}$ of the proximal jejunum. In LSG, the longitudinal resection of the stomach from the angle of His to approximately $5 \mathrm{~cm}$ proximal to the pylorus was performed using a 35 French bougie inserted along the lesser curvature. All operations were performed by the same team of surgeons.

\section{Chemicals, Instrumentation, and Analytical Methods}


All chemical reagents were of the highest grade available. Paracetamol, its internal standard metacetamol, and caffeine, paraxanthine and the internal standard diphylline, were from Sigma-Aldrich Quimica SA (Madrid, Spain). Plasma concentrations of all compounds were determined using a 1090 II high performance liquid chromatography modular system coupled to a 1100 Series UV detector (Agilent, Palo Alto, CA, USA). Caffeine and paraxanthine were extracted and analyzed following previously published methodology [16]. In a similar way, the simultaneous analysis of paracetamol and metacetamol in plasma was performed as reported elsewhere [17].

\section{Pharmacokinetics}

Data were fitted to one compartmental pharmacokinetic model with first-order elimination using GraphPad Prism (GraphPad Software, CA, USA, version 5.03 for Windows) and PKSolver (a freely available add-in program for Microsoft Excel). For further details, see the Supplementary Material.

\section{Statistics}

A descriptive analysis of both the baseline sociodemographic characteristics and the clinical parameters is given by the mean and the standard deviation for continuous variables and the absolute and relative frequencies for categorical ones. To compare the different weight categories (normal weight, overweight, and morbid obese) with respect to clinical parameters and plasma pharmacokinetic parameters of paracetamol, one-way ANOVA models were used. Statistical significance was set at 0.05 . The plasma pharmacokinetic parameters of paracetamol were analyzed as a function of time and treatment for obesity. For this purpose, repeated measures ANOVA models for the differences from baseline at sessions 2 and 3 were fitted for all parameters including time, treatment, and the interaction of both as factors. A detailed description of the statistical analyses can be found in the Supplementary Material. 


\section{Results}

\section{Baseline characteristics}

Anthropometric and biochemical parameters

The baseline characteristics of the 24 obese patients were compared with those of the normal weight $(n=14)$ and overweight $(n=14)$ volunteers. In our sample, the mean age of the obese subjects was higher than the mean age of normal and overweight ones. The obese group had a higher SBP compared to the normal and overweight groups. No statistically significant differences were found in DBP. The heart rate (HR) of obese individuals was higher than normal weight volunteers (Table 1).

\section{Paracetamol pharmacokinetics}

Paracetamol pharmacokinetic parameters in plasma following the oral intake of 500 mg of paracetamol (administered as a modified Karolinska cocktail) are summarized in Table 2 and plasma concentration-time profile is depicted in Figure 1. The maximum plasma concentration of paracetamol $\left(\mathrm{C}_{\max }\right)$ and its corresponding area under the curve (AUC) was significantly lower in morbid obese than in normal and overweight individuals $(\mathrm{p}<0.05)$. Paracetamol elimination half-life $\left(\mathrm{t}_{1 / 2}\right)$, time to reach $\mathrm{C}_{\max }\left(\mathrm{t}_{\max }\right)$, and elimination rate constant $\left(\mathrm{K}_{\mathrm{e}}\right)$ did not differ significantly among groups. The apparent volume of distribution $\left(\mathrm{V}_{\mathrm{D}} / \mathrm{F}\right)$ and metabolic clearance $(\mathrm{CL} / \mathrm{F})$ were greater in morbid obese than in normal and overweight subjects $(\mathrm{p}<0.005)$. However, after the individual correction for total body weight, neither $\mathrm{V}_{\mathrm{D}} / \mathrm{F}\left(\mathrm{V}_{\mathrm{D}} / \mathrm{F}\right.$.Weight) nor CL/F (CL/F.Weight) differed significantly among groups. These results are in agreement with data described in the literature [18].

Paraxanthine/caffeine (17X/137X) plasma levels 
Before surgery (Session 1) the obese group had lower concentrations of caffeine and paraxanthine compared to the normal and overweight groups $(\mathrm{p}<0.05)$. However, the ratio paraxanthine/caffeine did not differ significantly among groups (Table 3).

\section{Comparative effects between surgical procedures (LRYGR and LSG)}

\section{Clinical outcomes}

There were no serious perioperative complications related to either bariatric surgery or to secondary effects of the drugs used in the study. There was no mortality in either technique.

\section{Weight and BMI}

Figure 2 shows the evolution of total body weight and BMI at 1 and 6 months after bariatric surgery (session 2 and 3, respectively). As expected, four weeks after the intervention (session 2) a considerable reduction of body weight (mean loss of $-17 \mathrm{~kg}$ ) was observed $(\mathrm{p}<0.001)$.This reduction continued over time and a mean loss of $-32 \mathrm{~kg}$ was registered six months after surgery (Session 3) $(\mathrm{p}<0.001)$. A similar trend was observed in BMI. Despite finding marked differences among sessions none were observed between surgical procedures with respect to changes from baseline ( $\mathrm{p}=0.999)$ (Figure 2).

\section{Comparison of paracetamol pharmacokinetic parameters between surgical procedures}

No significant differences were found between LRYGB and LSG for any pharmacokinetic variable. Following bariatric surgery, an increase was observed in $\mathrm{AUC}_{0-4 \mathrm{~h}}$

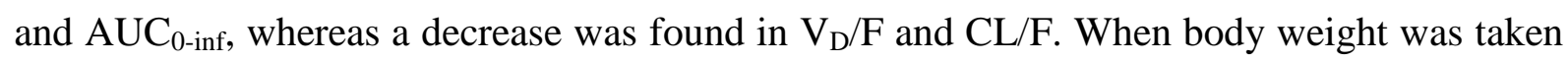

into account, the $\mathrm{V}_{\mathrm{D}} / \mathrm{F}$ did not differ significantly among sessions. $\mathrm{C}_{\max }$ did not change either among sessions, probably due to low statistical power (Supplementary Table 1). 
Comparison of plasma caffeine and paraxanthine concentrations between surgical procedures

No significant differences were found between LRYGB and LSG in plasma caffeine $(\mathrm{p}=0.616)$ and paraxanthine concentrations $(\mathrm{p}=0.903)$. In a similar way, the ratio paraxanthine/caffeine did not differ significantly between surgical techniques.

\section{Consequences of bariatric surgery: between sessions 1, 2 and 3}

\section{Paracetamol pharmacokinetic outcomes}

Paracetamol pharmacokinetic parameters changed as a consequence of bariatric surgery (Figure 3). An increase was observed in $\mathrm{C}_{\max }, \mathrm{AUC}_{0-4 \mathrm{~h}}$, and $\mathrm{AUC}_{0 \text {-inf, whereas a }}$ significant decrease was observed in $\mathrm{V}_{\mathrm{D}} / \mathrm{F}$ and $\mathrm{CL} / \mathrm{F}$. The assessment of relative bioavailability in sessions 2 and 3 compared with session 1 showed an increase in oral bioavailability in $\mathrm{C}_{\max }$ of $30 \%$ and $38 \%$, respectively, and an increase in $\mathrm{AUC}_{0 \text {-inf }}$ of $47 \%$ and $41 \%$, respectively. Distribution volume values of paracetamol did not present significant differences among all sessions after normalization with body weight. Additionally, nonrelevant differences in plasma clearance values of paracetamol, when normalized by body weight before and after 6 months of bariatric surgery, were observed. $T_{\max }$ values decreased from 1 hour (before surgery) to 0.5 hours ( 6 months after surgery), indicating a faster absorption as a consequence of the surgical intervention (Table 4).

\section{Caffeine and paraxanthine plasma concentrations}

Neither caffeine plasma concentrations nor the ratio paraxanthine/caffeine at 4 hours after a single oral administration of $65 \mathrm{mg}$ of caffeine differed significantly among sessions. However, in the case of paraxanthine, the plasma concentrations diminished at session 2 , becoming normalized at session 3 (Table 5). 


\section{Discussion}

In the present study we report that (1) paracetamol pharmacokinetics and caffeine plasma levels are altered in severely obese patients and (2) that two bariatric surgical techniques modify paracetamol oral bioavailability without impairing liver function (measured by CYP1A2 activity).

We have studied the impact of the two most commonly used bariatric surgery techniques (gastric bypass and sleeve gastrectomy) with regard to paracetamol pharmacokinetics (the most commonly used analgesic drug with a well-known pharmacokinetic profile) and caffeine metabolism (the most frequently consumed psychostimulant worldwide) [19]. It is important to evaluate paracetamol pharmacokinetics in obese subjects in order to determine whether the recommended dose for individuals with normal weight can be extrapolated to obese patients.

We found that paracetamol pharmacokinetics is altered in severe obesity. Morbid obese patients had a higher $\mathrm{V}_{\mathrm{D}}$ and $\mathrm{CL}$, and lower AUC and $\mathrm{C}_{\max }$ values, compared to normal weight and overweight individuals. When the apparent distribution volume and clearance were normalized by body weight, no differences were found, indicating that body weight was responsible for the apparent increase of these parameters. On the basis of our results, the recommended therapeutic doses of paracetamol are safe for obese individuals, as the plasma levels that are reached after an oral intake are lower than the ones achieved in normal and overweight individuals. In the case of therapeutic failure, the possibility of increasing the dose of paracetamol in obese patients in order to achieve plasma levels similar to those attained in normal weight individuals could be evaluated. Current guidelines for paracetamol dosage in obese patients recommend adjusting the dose of paracetamol using the ideal body weight [20]. Interestingly, after undergoing bariatric surgery, $\mathrm{V}_{\mathrm{D}}$ and $\mathrm{CL}$ values decreased and AUC and 
$\mathrm{C}_{\max }$ values increased, indicating a normalization of paracetamol pharmacokinetics. As an example, before surgery $\mathrm{AUC}_{0-4 h}$ was $32 \%$ lower in obese subjects than in normal weight individuals, but 6 months after surgery it was only $4 \%$ less. Moreover, $\mathrm{T}_{\max }$ value decreased from 1 hour (before surgery) to 30 minutes ( 6 months post-surgery). The normalization of the pharmacokinetic parameters can be explained by the considerable weight loss caused by bariatric surgery, whereas the decrease in $\mathrm{T}_{\max }$ is compatible with the consequence of the surgical procedure. Indeed, bariatric surgery leads to a faster entry of paracetamol to the intestine and its consequent absorption, as paracetamol absorption occurs mainly in the small intestine and strictly depends on the rate of gastric emptying $[4,6]$.

Taken together, these results indicate that after bariatric surgery paracetamol absorption takes place faster (earlier $\mathrm{T}_{\max }$ values), reaches higher plasma concentrations $\left(\mathrm{C}_{\max }\right)$, and results in a higher bioavailability (AUC) in comparison with the same individuals before surgery. It is noteworthy that normalization of paracetamol plasma levels was observed as a consequence of bariatric surgery as the AUC at 6 months after surgery did not differ from the bioavailability in normal weight individuals. On the basis of these results, we postulate that the increase in paracetamol oral bioavailability could be explained by a higher fraction of paracetamol absorbed across the gut wall (greater bioavailability) rather than by a higher rate of absorption. Indeed, when we employed the $\mathrm{C}_{\max } / \mathrm{AUC}_{0 \text {-inf }}$ ratio as an indicator of the rate of absorption [21, 22] we did not observe an increase in this parameter related to bariatric surgery (Table 4).

In the case of caffeine, obese patients had lower caffeine and paraxanthine plasma concentrations in comparison with normal and overweight individuals. This can be explained by the fact that the volume of distribution is markedly increased in obese subjects [23]. There were no baseline differences in the ratio paraxanthine/caffeine (17X/137X). Bariatric surgery did not alter either caffeine or the paraxanthine/caffeine ratio, indicating that CYP1A2 activity 
was unimpaired. A decrease in paraxanthine concentrations was observed 4 weeks after surgery but this was normalized at 6 months.

One of the strengths of the present study stems from its design, in which each subject acts as the corresponding control thus minimizing the influence of confounding variables and interindividual variability. Moreover, the inclusion of normal weight and overweight subjects allows the comparison of the values from morbid obese subjects with those of healthy ones. The present study has some limitations. Standard therapeutic doses of drugs were administered to study participants irrespective of their BMI. Another approach would be to adjust drug dosing by body weight. We believe that conclusions achieved would be the same following both dosing approaches. Although a prospective design was performed and we evaluated effects of bariatric surgery up to 6 months after surgery, we did not, however, assess the long-term effects when, according to the literature, some patients experience weight regain $[24,25]$. Neither did we include extremely morbid obese patients (BMI $>50$ or 60 ), nevertheless, the characteristics of our subjects concur with those in the vast majority of the articles published on bariatric surgery. Finally, it is worth mentioning that the Karolinska cocktail includes five drugs and this paper only focused on paracetamol and caffeine with a particular emphasis on their metabolism. The other drugs included in the cocktail (dextromethorphan, losartan, and omeprazole) and the impact of bariatric surgery on the activity of CYP isoforms are beyond the scope of this work and will be covered in a forthcoming article.

\section{Conclusions}

Paracetamol pharmacokinetics was altered in MO patients, presenting a higher apparent distribution volume and clearance, reaching lower plasma concentrations, and leading to lower bioavailability. As a consequence of bariatric surgery, absorption was faster 
and the previous parameters were normalized without differences being observed between both surgical procedures. In the case of caffeine, the paraxanthine/caffeine ratio was employed to study liver function. No differences were found in this ratio between obese patients and healthy individuals. Moreover, it was not altered by bariatric surgery, indicating that these bariatric techniques do not impair liver function assessed by CYP1A2 activity.

Conflict of interest disclosure: The authors declare that they have no conflict of interest.

Ethical statement: All procedures performed in the study involving human participants were in accordance with the ethical standards of the institutional and/or national research committee and with the 1964 Helsinki declaration and its later amendments or comparable ethical standards.

Consent statement: Informed consent was obtained from all individual participants included in the study. 


\section{References}

1. Stein J, Stier C, Raab H, Weiner R. Review article: the nutritional and pharmacological consequences of obesity surgery. Aliment Pharmacol Ther. 2014;40(6):582609.

2. Greenblatt HK, Greenblatt DJ. Altered Drug Disposition Following Bariatric Surgery: A Research Challenge. Clin Pharmacokinet. 2015;54(6):573-9.

3. Padwal R, Brocks D, Sharma AM. A systematic review of drug absorption following bariatric surgery and its theoretical implications. Obes Rev. 2010;11(1):41-50.

4. Prescott LF. Kinetics and metabolism of paracetamol and phenacetin. Brit J Clin Pharmacol. 1980;10(S2):291S-8S.

5. Raffa RB, Pergolizzi JV, Taylor R, Decker JF, Patrick JT. Acetaminophen (Paracetamol) Oral Absorption and Clinical Influences. Pain Practice. 2014;14(7):668-77.

6. Heading RC, Nimmo J, Prescott LF, Tothill P. The dependence of paracetamol absorption on the rate of gastric emptying. Br J Pharmacol. 1973;47(2):415-21.

7. Bartholomé R, Salden B, Vrolijk MF, Troost FJ, Masclee A, Bast A, et al. Paracetamol as a Post Prandial Marker for Gastric Emptying, A Food-Drug Interaction on Absorption. PLoS ONE. 2015;10(9):e0136618.

8. Renner E, Wietholtz H, Huguenin P, Arnaud MJ, Preisig R. Caffeine: A Model Compound for Measuring Liver Function. Hepatology. 1984;4(1):38-46.

9. Carrillo JA, Christensen M, Ramos SI, Alm C, Dahl M-L, Benítez J, et al. Evaluation of Caffeine as an In Vivo Probe for CYP1A2 Using Measurements in Plasma, Saliva, and Urine. Ther Drug Monit. 2000;22(4):409-17.

10. Doude van Troostwijk LJAE, Koopmans RP, Guchelaar H-J. Two novel methods for the determination of CYP1A2 activity using the paraxanthine/caffeine ratio. Fundam Clin Pharmacol. 2003;17(3):355-62. 
11. Hubbard V, Hall H. National Institutes of Health Consensus Development Conference Draft statement on gastrointestinal surgery for severe obesity. Obes Surg. 1991;1(3):257-65.

12. Benaiges D, Goday A, Pedro-Botet J, Mas A, Chillaron JJ, Flores-Le Roux JA. Bariatric surgery: to whom and when? Minerva Endocrinol. 2015 Jun;40(2):119-28.

13. Vidal P, Ramón JM, Goday A, Benaiges D, Trillo L, Parri A, et al. Laparoscopic Gastric Bypass Versus Laparoscopic Sleeve Gastrectomy as a Definitive Surgical Procedure for Morbid Obesity. Mid-Term Results. Obes Surg. 2013;23(3):292-9.

14. Christensen M, Andersson K, Dalén P, Mirghani RA, Muirhead GJ, Nordmark A, et al. The karolinska cocktail for phenotyping of five human cytochrome P450 enzymes. Clin Pharmacol Ther. 2003;73(6):517-28.

15. Hu OY-P, Tang H-S, Lane H-Y, Chang W-H, Hu T-M. Novel Single-Point Plasma or Saliva Dextromethorphan Method for Determining CYP2D6 Activity. J Pharmacol Exp Ther. 1998;285(3):955-60.

16. Carbó M, Segura J, De la Torre R, Badenas JM, Camí J. Effect of quinolones on caffeine disposition. Clin Pharmacol Ther. 1989;45(3):234-40.

17. Farré M, Roset P, Pascual J, Mas M, Sanagustín J, Menoyo E, et al. Estudio de la biodisponibilidad comparativa de la asociación de paracetamol y codeína (Gelocatil Codeína) frente a un preparado de referencia. Dolor. 1999;14:163-71.

18. Abernethy DR, Divoll M, Greenblatt DJ, Ameer B. Obesity, sex, and acetaminophen disposition. Clin Pharmacol Ther. 1982;31(6):783-90.

19. Cappelletti S, Daria P, Sani G, Aromatario M. Caffeine: Cognitive and Physical Performance Enhancer or Psychoactive Drug? Curr Neuropharmacol. 2015;13(1):71-88.

20. De Baerdemaeker LE, Mortier EP, Struys MM. Pharmacokinetics in obese patients. Contin Educ Anaesth Crit Care Pain. 2004;4(5):152-5. 
21. Portolés A, Puerro M, Terleira A, Rodríguez A, Caturla M-C, Fernández N, et al. A new high-absorption-rate Paracetamol 500-mg formulation: a comparative bioavailability study in healthy volunteers. Curr Ther Res. 2003;64(7):401-11.

22. Endrenyi L, Tothfalusi L. Metrics for the evaluation of bioequivalence of modifiedrelease formulations. AAPS J. 2012;14(4):813-9.

23. Abernethy DR, Todd EL, Schwartz JB. Caffeine disposition in obesity. Br J Clin Pharmacol. 1985;20(1):61-6.

24. Courcoulas AP, Christian NJ, Belle SH, Berk PD, Flum DR, Garcia L, et al. Weight Change and Health Outcomes at Three Years After Bariatric Surgery Among Patients with Severe Obesity. JAMA. 2013;310(22):2416-25.

25. Kushner RF, Sorensen KW. Prevention of Weight Regain Following Bariatric Surgery. Curr Obes Rep. 2015;4(2):198-206. 


\section{Figure legends}

Figure 1. Basal (Session 1) time-course plasma concentrations of paracetamol after the oral administration of $500 \mathrm{mg}$ of paracetamol (administered as a modified Karolinska cocktail) to normal weight $(n=14)$, overweight $(n=14)$ and morbid obese $(n=24)$ volunteers. Data are expressed mean \pm SEM.

Figure 2. Evolution of body weight (A) and body mass index (BMI; B) before surgery (session 1), four weeks post-surgery (session 2), and six months post-surgery (session3). Values are expressed as mean $\pm S D$.

Figure 3. Time-course plasma concentrations of paracetamol following the oral administration of $500 \mathrm{mg}$ of paracetamol (administered as a modified Karolinska cocktail) in morbid obese volunteers before (session 1), one month (session 2), and six months (session 3) after bariatric surgery. Data are expressed mean \pm SEM. 


\section{Tables}

Table 1. Sociodemographic baseline characteristics and basal clinical parameters of the 24 patients, compared with normal weight and overweight volunteers. Data are expressed as mean $\pm S D . *$ Significant differences between the three subject groups. NS: Not significant

\begin{tabular}{|ccccc|}
\hline N & Normal weight & Overweight & Obese & *Differences \\
\hline Age (years) & 14 & 14 & 24 & - \\
\hline Females & $32.2 \pm 5.6$ & $34.6 \pm 6.6$ & $41.4 \pm 8.8$ & *Normal, overweight < obese \\
\hline Males & 2 & 14 & 24 & - \\
\hline Smokers & $36 \%$ & 0 & 0 & - \\
\hline Height (cm) & $167 \pm 9$ & $161 \pm 5$ & $161 \pm 9$ & - \\
\hline Weight (kg) & $64.4 \pm 7.6$ & $71.4 \pm 5.4$ & $111.6 \pm 15.0$ & *Normal < overweight < obese \\
\hline BMI (kg/m $\left.{ }^{2}\right)$ & $23.0 \pm 1.2$ & $27.6 \pm 1.1$ & $43.1 \pm 3.4$ & *Normal < overweight < obese \\
\hline SBP (mm Hg) & $107.3 \pm 7.6$ & $114.9 \pm 9.9$ & $124.3 \pm 12.3$ & *Normal < overweight < obese \\
\hline DBP (mm Hg) & $66.6 \pm 6.2$ & $65.6 \pm 6.9$ & $68.8 \pm 10.7$ & \\
\hline HR (bpm) & $61.4 \pm 8.5$ & $65.9 \pm 7.0$ & $68.4 \pm 8.5$ & *Normal < obese, overweight \\
\hline
\end{tabular}


Table 2. Plasma pharmacokinetic parameters of paracetamol in normal weight $(n=14)$, overweight $(n=14)$, and obese $(n=24)$ volunteers after a single oral administration of modified Karolinska cocktail containing 500 mg of paracetamol. Data are expressed as mean $\pm S D$ with the only exception of $t_{\max }$, where median and minimum-maximum ranges are given. *Significant differences between the three subject groups. NS: Not significant.

\begin{tabular}{|c|c|c|c|c|}
\hline & $\begin{array}{c}\text { Normal } \\
\text { weight }\end{array}$ & Overweight & Obese & *Differences \\
\hline$C_{\max }(\mu g / m L)$ & $7.0 \pm 1.7$ & $7.6 \pm 1.8$ & $5.4 \pm 2.3$ & *Normal, overweight $>$ obese \\
\hline $\mathrm{AUC}_{0-4 h}(\mu \mathrm{g} \cdot \mathrm{h} / \mathrm{mL})$ & $16.4 \pm 3.3$ & $16.9 \pm 3.9$ & $11.2 \pm 4.7$ & *Normal, overweight $>$ obese \\
\hline $\operatorname{AUC}_{0-\text { inf }}(\mu \mathrm{g} \cdot \mathbf{h} / \mathbf{m L})$ & $24.4 \pm 5.6$ & $23.8 \pm 5.5$ & $15.5 \pm 6.7$ & *Normal, overweight $>$ obese \\
\hline $\mathbf{K}_{\mathrm{e}}\left(\mathbf{h}^{-1}\right)$ & $0.36 \pm 0.09$ & $0.37 \pm 0.06$ & $0.42 \pm 0.11$ & NS \\
\hline $\mathbf{t}_{1 / 2}(\mathbf{h})$ & $2.07 \pm 0.58$ & $1.91 \pm 0.36$ & $1.76 \pm 0.43$ & NS \\
\hline $\mathbf{t}_{\max }(h)$ & $1(0.5-3)$ & $1(0.5-2)$ & $1(0.5-2)$ & NS \\
\hline $\mathbf{V}_{\mathbf{D}} / \mathbf{F}(\mathbf{L})$ & $62.3 \pm 16.7$ & $60.0 \pm 13.8$ & $90.8 \pm 26.1$ & $*$ Normal, overweight $<$ obese \\
\hline $\mathrm{V}_{\mathrm{D}} / \mathrm{F}$.Weight (L/kg) & $0.96 \pm 0.20$ & $0.83 \pm 0.15$ & $0.81 \pm 0.20$ & NS \\
\hline CL/F (L/h) & $21.5 \pm 4.6$ & $22.3 \pm 5.7$ & $38.7 \pm 17.9$ & *Normal, overweight < obese \\
\hline CL/F.Weight (L/h/kg) & $0.33 \pm 0.07$ & $0.31 \pm 0.06$ & $0.35 \pm 0.17$ & NS \\
\hline
\end{tabular}


Table 3. Caffeine and paraxanthine plasma concentrations in normal weight $(n=14)$, overweight $(n=14)$, and obese $(n=24)$ volunteers 4 hours after a single oral administration of modified Karolinska cocktail containing 60 mg of caffeine. Data are expressed as mean $\pm S D$. *Significant differences between the three subject groups. NS: Not significant

\begin{tabular}{|ccccc|} 
& Normal weight & Overweight & Obese & *Differences \\
\hline Caffeine (ng/mL) & $0.32 \pm 0.16$ & $0.36 \pm 0.18$ & $0.23 \pm 0.11$ & $*$ Normal, overweight > obese \\
\hline Paraxanthine (ng/mL) & $0.11 \pm 0.04$ & $0.10 \pm 0.03$ & $0.08 \pm 0.03$ & $*$ Normal, overweight > obese \\
\hline $\mathbf{1 7 X / 1 3 7 X ~ ( m o l a r ~ r a t i o ) ~}$ & $0.40 \pm 0.12$ & $0.37 \pm 0.18$ & $0.42 \pm 0.21$ & NS \\
\hline
\end{tabular}


Table 4. Plasma pharmacokinetic parameters of paracetamol following the oral administration of $500 \mathrm{mg}$ of paracetamol in obese patients ( $n=24)$ before (session 1), one month (session 2), and six months (session 3) after undergoing bariatric surgery. Data are expressed as mean $\pm S D$ with the only exception of $t_{\max }$, where median and minimummaximum ranges are given. *Significant differences between the three sessions in the morbid obese subjects. NS: Not significant.

\begin{tabular}{|c|c|c|c|c|}
\hline & Session 1 & Session 2 & Session 3 & *Differences \\
\hline$C_{\max }(\mu \mathrm{g} / \mathrm{mL})$ & $5.36 \pm 2.29$ & $6.99 \pm 2.75$ & $7.39 \pm 2.60$ & $\mathrm{~S} 1<\mathrm{S} 2, \mathrm{~S} 3$ \\
\hline $\mathrm{AUC}_{0-4 \mathrm{~h}}(\mu \mathrm{g} \cdot \mathbf{h} / \mathbf{m L})$ & $11.2 \pm 4.7$ & $15.3 \pm 5.1$ & $15.8 \pm 3.6$ & $\mathrm{~S} 1<\mathrm{S} 2, \mathrm{~S} 3$ \\
\hline $\operatorname{AUC}_{0 \text {-inf }}(\mu \mathrm{g} \cdot \mathbf{h} / \mathbf{m L})$ & $15.5 \pm 6.7$ & $22.8 \pm 8.5$ & $21.8 \pm 6.5$ & $\mathrm{~S} 1<\mathrm{S} 2, \mathrm{~S} 3$ \\
\hline $\mathbf{k}_{\mathrm{e}}\left(\mathbf{h}^{-1}\right)$ & $0.42 \pm 0.11$ & $0.34 \pm 0.08$ & $0.38 \pm 0.09$ & $\mathrm{~S} 1>\mathrm{S} 2<\mathrm{S} 3$ \\
\hline $\mathbf{t}_{1 / 2}(\mathbf{h})$ & $1.76 \pm 0.43$ & $2.17 \pm 0.47$ & $1.91 \pm 0.42$ & $\mathrm{~S} 1<\mathrm{S} 2>\mathrm{S} 3$ \\
\hline $\mathbf{t}_{\max }(h)$ & $1(0.5-2.0)$ & $1(0.5-2.0)$ & $0.5(0.25-1.0)$ & $\mathrm{S} 2, \mathrm{~S} 1>\mathrm{S} 3$ \\
\hline $\mathbf{V}_{\mathbf{D}} / \mathbf{F}(\mathbf{L})$ & $90.8 \pm 26.1$ & $75.2 \pm 23.0$ & $65.4 \pm 11.3$ & $\mathrm{~S} 1>\mathrm{S} 2, \mathrm{~S} 3$ \\
\hline $\mathrm{V}_{\mathrm{D}} / \mathrm{F}$. Weight (L/kg) & $0.81 \pm 0.20$ & $0.79 \pm 0.18$ & $0.83 \pm 0.13$ & NS \\
\hline CL/F (L/h) & $38.7 \pm 17.9$ & $25.8 \pm 12.9$ & $25.1 \pm 8.1$ & $\mathrm{~S} 1>\mathrm{S} 2, \mathrm{~S} 3$ \\
\hline CL/F.Weight (L/h/kg) & $0.35 \pm 0.17$ & $0.27 \pm 0.12$ & $0.32 \pm 0.11$ & $\mathrm{~S} 1>\mathrm{S} 2<\mathrm{S} 3$ \\
\hline Relative bioavailability $\left(\mathrm{F}_{\text {rel }}\right)$ & 100 & $160.8 \pm 49.3$ & $149.6 \pm 39.8$ & $\mathrm{~S} 1<\mathrm{S} 2, \mathrm{~S} 3$ \\
\hline $\mathrm{C}_{\text {max }} / \mathrm{AUC}_{\mathbf{0 - \text { inf }}}\left(\mathrm{h}^{-1}\right)$ & $0.36 \pm 0.09$ & $0.32 \pm 0.10$ & $0.36 \pm 0.14$ & NS \\
\hline
\end{tabular}


Table 5. Caffeine and paraxanthine plasma concentrations in obese patients $(n=24)$ before (session 1), one month (session 2), and six months (session 3) after undergoing bariatric surgery. Concentrations were determined 4 hours after a single oral administration of modified Karolinska cocktail containing $65 \mathrm{mg}$ of caffeine. Data are expressed as mean \pm SD. *Significant differences between the three sessions in the morbid obese subjects. NS: Not significant.

\begin{tabular}{|ccccc|}
\hline Caffeine (ng/mL) & Session 1 & Session 2 & Session 3 & *Differences \\
& $0.21 \pm 0.09$ & $0.21 \pm 0.08$ & $0.24 \pm 0.12$ & NS \\
\hline Paraxanthine (ng/mL) & $0.07 \pm 0.03$ & $0.05 \pm 0.02$ & $0.08 \pm 0.04$ & S1>S2<S3 \\
\hline Paraxanthine/Caffeine & $0.44 \pm 0.22$ & $0.38 \pm 0.35$ & $0.48 \pm 0.33$ & NS \\
\hline
\end{tabular}

